\title{
Research on ADS1282-based Distributed Seismic Data Acquisition System
}

\author{
H.M. Wang, W.B. Wei, M. Deng, Q.S. Zhang, J.Y. Huang \\ College of Geophysics and Information Technology \\ Key Laboratory of Underground Information Detection Technology and Instrument Education Department \\ China University of Geosciences \\ Beijing, China
}

\begin{abstract}
The distributed seismic data acquisition system is a critical component to telemetry digital seismograph, while the performance of digital seismograph is subject to the performance index of this data acquisition system. A distributed seismic data acquisition system with high accuracy and low power dissipation which is applicable to large scale wired digital telemetry seismograph was developed with high accurate analog-digital conversion technology, FPGA embedded technology and low power consumption technology in this paper. This system qualifies A class standard specified in SY/T5391-2007 in the aspects of equivalent input noise, harmonic distortion, dynamic range with unique features of low power consumption and low cost.
\end{abstract}

Keywords-seismic data acquisition system; high accuracy acquisition; low power consumption

\section{INTRODUCTION}

The petroleum consumption volume and crude oil import volume in our country are increasing as the national economy kept growing by years, while the foreign-trade dependence is increasing as well. According to the present national economy and the growth tendency in petroleum consumption, the foreign trade dependence in petroleum by 2020 would increase from $62 \%$ in 2012 to $67 \%$ [1]. In order to maintain the safe and sustainable national economy development, it is necessary to expedite the exploration of petroleum reserves and the location of new reserves in our country, promote the capability to explore and develop petroleum by ourselves, and enhance the independent ability and innovation in petroleum equipments.

The multiple channel digital telemetry seismograph, which is widely used in precise exploration in petroleum, natural gas, and coal recourses, is one of the typical seismographs at present. This product is featured in strong channel capability, high performance to reject interference, small system mass, low in power dissipation, high operation efficiency, and allow real time acquisition [2]. Multiple channel wired digital telemetry seismograph is generally consist of seismometer, acquisition system (FDU), power system (LAUL), crossing system (LAUX), apparatus truck as well as other devices[3]. As the mote significant end in telemetry seismic data acquisition system, distributed seismic data acquisition system is one of the critical components; the integral performance of telemetry seismic data acquisition system shall depend on the performance index of this individual component. A distributed seismic data acquisition system with high accuracy and low power dissipation which is applicable to large scale wired digital telemetry seismograph was developed with high accurate analog-digital conversion technology, FPGA embedded technology and low power consumption technology in this paper.

\section{BASIC STRUCTURE OF SEISMIC DATA ACQUISITION SYSTEM}

The requirements on key indicators in seismic data acquisition system such as the resolution, dynamic range, signal-to-noise ratio, channel capacity, power consumption and portability are stricter as the scientific technology grows rapidly $[3,4,5]$.

The functional block diagram of distributed seismic data acquisition system designed based on ADS1282 and DAC 1282 in this paper is as in Fig.1. The hardware circuit is mainly consisted of data acquisition circuit, master control circuit based on FPGA master chip and peripheral circuit and power circuit.

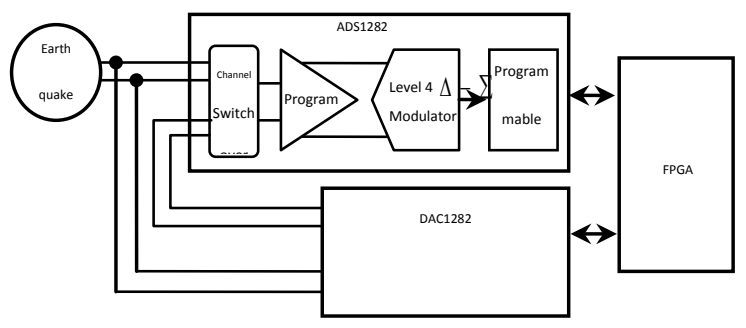

FIGURE I. CHART OF SEISMIC DATA ACQUISITION SYSTEM BASED ON ADS1282 AND DAC1282.

The earth vibration caused by seismic wave would convert by seismometer into electric signal; the data acquisition circuit would carry out the digitalization of seismic signal as well as the packing, proposal, storage and retransmission of data. The system would transfer the digital signal to FPGA master controller for operation and process. DAC1282 would serve as seismic test signal generator to provide signal for system test.

FPGA mater control circuit would transmit control command to data acquisition circuit and provide logic control level for relevant chip pins so as to set the sampling frequency 
and sampling length required for data acquisition; arrange front transmission gain; switch operation modes and input channel; receive analyze and forward the data frames from power source station.

The power circuit shall acquire power from transmission cable at first and then perform voltage convert to export satisfied stable power voltage for data acquisition circuit and FPGA master control circuit.

\section{DATA ACQUISITION CIRCUIT BASED ON ADS1282 AND DAC1282}

The data acquisition circuit board adopt a six layer structure which are: top signal layer, simulate ground layer, signal layer, power layer, reference ground and digital ground layer and bottom signal layer. Layers would connect through holes.

The high precision analog-digital converter ADS1282 and total integration digital analog converter suitable for seismic test DAC1282 [6] produced by Texas Instruments make it possible to simply the complicated circuit design and reduce the power dissipation. DAC1282 could serve as seismic test signal generator and realize the pulse, THD and common mode rejection ratio from DAC output end to seismometer input and channel test in ADS1282 input by control the change-over switch. The master clock input is $4.096 \mathrm{MHz}$ which is generated from oscillator. In order to keep the master clock synchronize with the clock in ADS1282, those two clocks share one clock source.

The low noise programmable gain amplifier (PGA) integrated in ADS1282 is featured in excellent low noise $(5 \mathrm{nV} \sqrt{H Z})$ and high input impedance[7,8], which allows the seismometer to connect with front gains of $\times 1, \times 4, \times 8, \times 16$, $\times 64$; two difference channels would connect with DAC1282 and front circuit. The power supply applies double electrodes power, the analog power would be $\pm 2.5 \mathrm{~V}$, the digital power would be $3.3 \mathrm{~V}$, the external reference voltage would be $+2.5 \mathrm{~V}$.

FPGA master control circuit could realize the function of data acquisition control, data process, communication transmission as well as other functions, considering the system dissipation, user I/O number requirements and logic resource demands, EP4CE6E22I7N with the lest demand in logic resource and $\mathrm{I} / \mathrm{O}$ resource and lowest power dissipation in Cyclone IV E series was adopted in this design, a double-layer structure was selected to satisfy the requirements in internal space and shape of data acquisition system. FPGA could control ADS1282 via SPI interface consisted of SCLK, MISO, MOSI and DRDY. In addition, external synchronizing signal input SYNC and signal over frame signal output MFLAG are also provided in this circuit.

Distributed seismic data acquisition system would acquire power from four core 408UL master cable, the master cable could transfer data and supply acquisition system with direct current which is critical to the low power dissipation of this data acquisition system, in order to satisfy the various demand of different chips, the power circuit design adopts a series of low power dissipation voltage switching circuits which could switch $48 \mathrm{~V}$ to $+3.3 \mathrm{~V},+3.3 \mathrm{~V}$ to $+1.2 \mathrm{~V}$ (or $+1.1 \mathrm{~V})$ and $+2.5 \mathrm{~V}$,
$+3.3 \mathrm{~V}$ to +2.5VA and -2.5VA. Meanwhile the power isolation between the acquisition system and power system could be realized with the application of low dissipation isolation flyback converter LT8300ES5, thus to avoid the interference of electric signal and the ground isolation with noise source voltage. The maximum output current in this circuit is $440 \mathrm{~mA}$ which could meet the power demand from the digital circuit in distributed seismic data acquisition system.

\section{ADS1282 AND DAC1282 DRIVER PROGRAM DESIGN}

FPGA would control ADS1282 and DAC1282 separately via two independent SPI interfaces. The development and design of software program in this paper shall subject to the actual function requirements and completed with VHDL hardware description language in Quartus II integrated platform.

In order to ensure the normal operation of ADS1282, the operation sequence is as follows:

1) Reset;

2) Initialize sampling rate, channel gains as well as other parameters;

3) Export synchronizing signal SYNC;

4) Wait data to prepare signal $\overline{D R D Y}$, read the converted 32 bit digit after the failing edge of $\overline{D R D Y}$.

The operation sequence of DAC1282 is as follows:

1) Select SW mode, switch among Sine, DC and Pulse test modes;

2) Export synchronizing signal SYNC;

3) Arrange the output amplitude of analog signal and operation mode.

ADS1282 and DAC1282 have the same SPI write register timing sequence. The command to write register is consist of two byte operation code with one or more register data awaiting to be written, the format of first operation code is "0010rrrr", in which rrrr represent the initial address of register to be written; the format of second operation code is "0000nnnn", in which nnnn represent -1 to be written in register.

\section{SEISMIC DATA ACQUISITION SYSTEM PERFORMANCE TEST}

The test on the performance and power dissipation of acquisition system were carried out when the hardware and software design of the system were completed. The performance tests include equivalent input noise test, harmonic distortion test, dynamic range test as well as other relevant tests. The test platform consists of DC, seismic data acquisition system and PC installed with test software (Quartus II and Matlab).

\section{A. Equivalent Input Noise Test}

A terminal resistance should be connected to analog signals in equivalent input noise test, CS3301A and ADS1282 could switch the input mode to use the build-in $400 \Omega$ terminal 
resistance in noise test. In the course of test, the sampling ratio of $\mathrm{A} / \mathrm{D}$ convertor is $1000 \mathrm{SPS}$, the sampling time is $2 \mathrm{~s}$, process and analyze the conversion data when the front gains in sampling is $\times 1, \times 4, \times 8, \times 16, \times 64$; apply multiple measures and take an average by the time of calculation.

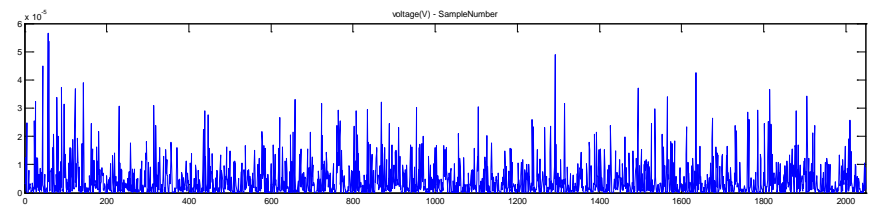

FIGURE II. EQUIVALENT INPUT NOISE CURVE.

The equivalent input noise curve as in Fig.2 was obtained after process on 2048 input noise sampling points (front gain $\times 1$ ). The equivalent input noise is $1.1822 \mu V$, which is superior to A class standard (1.6 $\mu V$ ) specified in SY/T53912007.

\section{B. Harmonic Distortion Test}

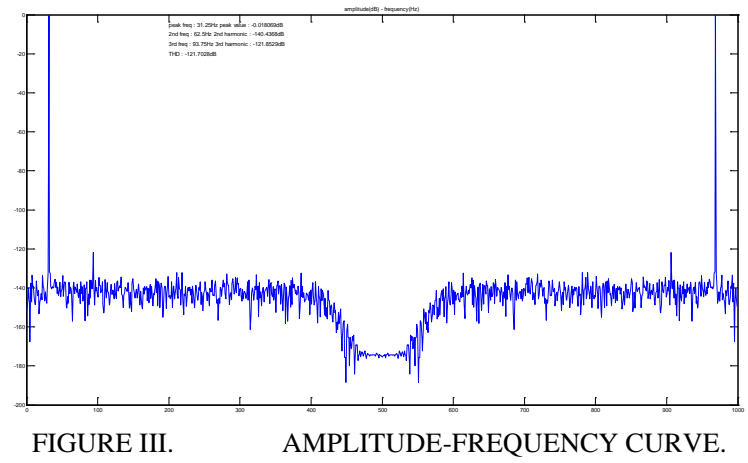

By the time of test, the system is equipped with full amplitude sine wave with DAC export frequency of $31.25 \mathrm{~Hz}$, the front gains is $\times 1$, the sampling rate of $A / D$ convertor is 1000SPS, the sampling time is 2s. The converted data would be exported by CSV format and then processed and analyzed with Matlab software. The harmonic distortion value of acquisition layer is $-121.7 \mathrm{~dB}$ which qualifies A class standard in SY/T5391-2007 (less than -106dB) and satisfies the requirements in seismic data acquisition.

\section{Acquisition System Power Dissipation Test}

Distributed seismic data acquisition system with lower power dissipation is one of the main purposes of this paper. Acquisition system power dissipation test was performed in order to clarify the power dissipation features of distributed seismic data acquisition system. The quiescent dissipation and operation dissipation of data acquisition circuit, FPGA master control circuit and the entire acquisition system were tested. The test results of single layer power dissipation of acquisition board and master control board is as follows in table 1.
TABLE I. TEST RESULTS OF SINGLE LAYER POWER DISSIPATION OF ACQUISITION LAYER AND MASTER CONTROL LAYER.

\begin{tabular}{c|c|c|c|c}
\hline Test object & $\begin{array}{c}\text { Cirrus } \\
\text { acquisition } \\
\text { board }\end{array}$ & $\begin{array}{c}\text { ADS1282 } \\
\text { acquisition } \\
\text { board }\end{array}$ & $\begin{array}{c}\text { Cyclone VI } \\
\text { master } \\
\text { control } \\
\text { board }\end{array}$ & $\begin{array}{c}\text { Cyclone V } \\
\text { master } \\
\text { control } \\
\text { board }\end{array}$ \\
\hline $\begin{array}{c}\text { Quiescent } \\
\text { dissipation } \\
(\text { mW) }\end{array}$ & 75.9 & 89.1 & 115.5 & 217.8 \\
\hline $\begin{array}{c}\text { Operation } \\
\text { dissipation } \\
\text { (mW) }\end{array}$ & 188.1 & 138.6 & 135.3 & 283.8 \\
\hline
\end{tabular}

\section{Dynamic Range Test}

The dynamic range could be obtained by the following formula.

$$
D R=20 \lg (E / n)
$$

By the time of test, the sampling ratio of $A / D$ convertor is 1000 SPS, front gain $\times 1$, sampling time is 2 s. Calculate RMS and $n$ with the converted value of maximum output signal in channel and non-driving signal, thus to obtain the dynamic range of acquisition layer of $121.7 \mathrm{~dB}$ which qualify A class standard in SY/T5391-2007 (110dB).

\section{CONCLUSION}

In this paper, ADS1282 high precision seismic exploration purpose chip is applied in the design of distributed seismic data acquisition system, and the simulation acquisition accuracy in aspects of front end circuits, power circuits and PCB board design is guaranteed. FPGA chip complies with resource and power consumption requirements. Through a series of experiments, the tests for acquisition performance such as acquisition system equivalent input noise, harmonic distortion and dynamic range are finished, and the static and dynamic power consumption of acquisition board, master control board and entire acquisition system. In accordance with test results, the performances of data acquisition circuit in aspects of equivalent input noise, harmonic distortion and dynamic range are outstanding, and all reach Class A standard in SY/T5391-2007. It has the most excellent low power consumption characteristics and minimum costs.

\section{ACKNOWLEDGEMENTS}

This work is supported by the National Natural Science Foundation of China (No.41204135), the National "863" Program of China (No.2012AA061102), the Fundamental Research Funds for the Central Universities of China (No.2652011262), and the Fundamental Research Funds for the Central Universities of China (No.35932011295).

\section{REFERENCES}

[1] Tian Chunrong, Analysis on Export and Import Status of Petroleum and Natural Gas in China in 2012, International Petroleum Economics, 3: 44 55, 2013.

[2] Cheng Jianyuan, Wang Pan, Wu Hai, Development Process and Trend of Seismic Exploration Instrument, Coal Science and Technology, 41 (1): 30 35, 2013. 
[3] Zhang Qishen, Zhang Qimao, Guo Jian, Application of Embedded Linux Technology to Telemetering Digital Seismic Power Station, Geosciences, 26 (6): 1322 1326, 2012.

[4] Xu Shanhui, Guo Jian, Luo Weibing, Recovery Study on 24-Digit Telemetering Digital Seismograph Test Record, Equipment for Geophysical Prospecting, 22 (1): 6 9, 2012.

[5] Zhang Qisheng, Deng Ming, Cui Jinling, et al. Research and development of one novel distributed digital seismic acquisition station, Advances in Information Sciences and Service Sciences, 4(5):184 190, 2012.

[6] Texas Instruments Incorporated. DAC1282 datasheet, Texas Instruments Incorporated, 2012.

[7] Texas Instruments Incorporated. ADS1282 datasheet, Texas Instruments Incorporated, 2013.

[8] Altera Corporation, Cyclone IV Device Handbook, Altera Corporation, 2013. 